\title{
Rényi entropy dynamics and Lindblad spectrum for open quantum systems
}

\author{
Yi-Neng Zhou $\odot,{ }^{1, *}$ Liang Mao $\odot,{ }^{1,2, *}$ and Hui Zhai $\odot^{1, \dagger}$ \\ ${ }^{1}$ Institute for Advanced Study, Tsinghua University, Beijing 100084, China \\ ${ }^{2}$ Department of Physics, Tsinghua University, Beijing 100084, China
}

(Received 8 February 2021; accepted 7 October 2021; published 21 October 2021)

\begin{abstract}
In this paper we point out that the Lindblad spectrum of a quantum many-body system displays a segment structure and exhibits two different energy scales in the strong dissipation regime. One energy scale proportional to the dissipation strength determines the separation between different segments, and the other energy scale inversely proportional to the dissipation strength determines the broadening of each segment. Utilizing an approximate relation between the dynamics of the second Rényi entropy and the Lindblad spectrum, we show that these two energy scales respectively determine the short-time and long-time dynamics of the second Rényi entropy starting from a generic initial state. This gives rise to opposite behaviors between the short-time and long-time dynamics. That is, as the dissipation strength increases, the short-time dynamics become fast, and the long-time dynamics become slow. We also interpret the quantum Zeno effect as the specific initial states that only occupy the Lindblad spectrum around zero. Therefore, only the broadening energy scale of the Lindblad spectrum matters in the entropy dynamics. For this reason, the entropy dynamics are suppressed as the dissipation increases. To concretely illustrate these results, two models that can be verified experimentally have been studied numerically.
\end{abstract}

DOI: 10.1103/PhysRevResearch.3.043060

\section{INTRODUCTION}

For a closed quantum system, the energy spectrum of its Hamiltonian fully determines the timescales of its dynamics. For an open quantum system, when the environment is treated as Markovian, the couplings between the system and environment are controlled by a set of dissipation operators. In this case, the dynamics of the system are governed by the Lindblad master equation which contains the contributions from both the Hamiltonian and the dissipation operators [1]. The spectrum of the Hamiltonian alone can no longer determine the timescales of the entire dynamics, and a natural question is then what energy scales set the timescales of dynamics of an open quantum system.

There are various directions to approach this issue, and the answer also relies on what type of dynamics we are concerned with. Here let us focus on the dissipation-driven dynamics. There are still different physical intuitions from different perspectives. One intuition is from the perturbation theory when the dissipation strength is weaker compared with the typical energy scales of the Hamiltonian [2]. In this regime, treating the dissipation perturbatively gives rise to a scenario in which the dissipation dynamics become faster when the dissipation

\footnotetext{
*These authors contributed equally to this work.

†hzhai@tsinghua.edu.cn
}

Published by the American Physical Society under the terms of the Creative Commons Attribution 4.0 International license. Further distribution of this work must maintain attribution to the author(s) and the published article's title, journal citation, and DOI. strength is stronger. Another intuition is from the studies of the quantum Zeno effect [3-6] which states that frequent measurements can slow down the dynamics provided that the typical time interval between two successive measurements is shorter than the intrinsic timescale of the system. Since the measurement can also be understood in terms of dissipations in the Lindblad master equation, it provides another scenario in which the dissipation dynamics is suppressed when the dissipation becomes stronger when the dissipation strength is stronger than the typical energy scales of the Hamiltonian. It seems that these two scenarios respectively apply to different parameter regimes, and the results are also qualitatively opposite to each other. It will be interesting to see that there exists a framework that can unify these two scenarios.

When a system is coupled to a Markovian environment, the entropy of the system will increase over time. The entropy dynamics of an open quantum many-body system is a subject that has attracted lots of interest recently [7-13]. In this paper we address the issue of typical timescales of the entropy increasing dynamics of a quantum many-body system coupled to a Markovian environment. Especially, we focus on the second Rényi entropy for the reason that will be clear below, and we will answer the question of whether the entropy dynamics becomes faster or slower when the dissipation strength increases. The main results of this work are summarized as follows:

(i) We consider the strong dissipation regime where the dissipation strength is stronger than the typical energy scales of the Hamiltonian. We show that the Lindblad spectrum of a quantum many-body system displays a segment structure and exhibits two different energy scales. The first energy scale proportional to the dissipation strength determines the 


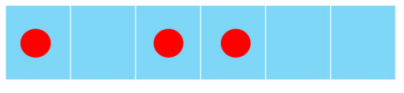

$i \hbar \frac{\partial \hat{\rho}}{\partial t}=\hat{\mathcal{L}} \hat{\rho}$

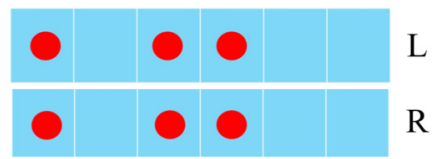

$i \hbar \frac{\partial \Psi_{\rho}}{\partial t}=\left(\hat{H}_{S}-i \hat{H}_{D}\right) \Psi_{\rho}$
FIG. 1. Schematic of the mapping between the Lindblad master equation (left) and the Schödinger-like equation in a doubled system (right). Here $\hat{L} \hat{\rho}$ denotes the r.h.s. of Eq. (1).

separation between different segments, and the second energy scale inversely proportional to the dissipation strength determines the broadening of each segment.

(ii) For generic initial state, the first energy scale determines the short-time dissipation dynamics, and the second energy scale determines the long-time dissipation dynamics. As a result, when the dissipation strength increases, the short-time dynamics become fast, and the long-time dynamics become slow.

(iii) For a certain specific initial state that only occupies the lowest segment, only the second energy scale enters the dynamics. As a result, the dynamics always become slow when the dissipation strength increases, giving rise to the quantum Zeno effect.

\section{LINDBLAD IN DOUBLED SPACE}

Our studies are based on a mapping between the Lindblad master equation and a nonunitary evolution of wave function in a doubled space, as shown in Fig. 1. This mapping is called vectorization or Choi-Jamiolkwski isomorphism in literature.

Let us first review this mapping [14-16]. Considering a density matrix $\hat{\rho}$, and given a set of complete bases $\{|n\rangle\}(n=$ $1, \ldots, \mathcal{D}_{\mathrm{H}}$ ), of the Hilbert space with dimension $\mathcal{D}_{\mathrm{H}}$ (say the eigenstates of the Hamiltonian $\hat{H}$ with eigenenergies $E_{n}$ ), the density matrix $\hat{\rho}$ can be expressed as $\hat{\rho}=\sum_{m n} \rho_{m n}|m\rangle\langle n|$. By this operator-to-state mapping, we can construct a wave function $\Psi_{\rho}=\sum_{m n} \rho_{m n}|m\rangle \otimes|n\rangle$ which contains exactly the same amount information as $\hat{\rho}$ does. Here $\Psi_{\rho}$ is a wave function on a system whose size is doubled compared with the original system, and we will refer these two copies of original system as the "left" (L) and the "right" (R) systems. For instance, a density matrix of a pure state $\hat{\rho}=$ $|\psi\rangle\langle\psi|$ is mapped to a product state $\Psi_{\rho}=|\psi\rangle \otimes|\psi\rangle$ in the double system Under this mapping, and a thermal density matrix $\hat{\rho}=\sum_{n} e^{-E_{n} /\left(k_{\mathrm{b}} T\right)}|n\rangle\langle n|$ at temperature $T$ is mapped to a thermofield double state at temperature $T / 2$ as $\Psi_{\rho}=$ $\sum e^{-E_{n} /\left(k_{\mathrm{b}} T\right)}|n\rangle \otimes|n\rangle$.

For an open system coupled to a Markovian environment, the dynamics of the density matrix obey the Lindblad master equation given by

$$
\hbar \frac{d \hat{\rho}}{d t}=-i[\hat{H}, \hat{\rho}]+\sum_{\mu} \gamma_{\mu}\left(2 \hat{L}_{\mu} \hat{\rho} \hat{L}_{\mu}^{\dagger}-\left\{\hat{L}_{\mu}^{\dagger} \hat{L}_{\mu}, \hat{\rho}\right\}\right),
$$

where $\hat{L}_{\mu}$ stand for a set of dissipation operators, and $\gamma_{\mu}$ are their corresponding dissipation strengths. In this work we consider the situations where all $\hat{L}_{\mu}$ are Hermitian. After the mapping, the wave function $\Psi_{\rho}$ in the double system satisfies a Schrödinger-like equation

$$
i \hbar \frac{d \Psi_{\rho}}{d t}=\left(\hat{H}_{\mathrm{s}}-i \hat{H}_{\mathrm{d}}\right) \Psi_{\rho} .
$$

Here $\hat{H}_{\mathrm{s}}$ is the Hermitian part of the Hamiltonian determined by the system itself, and it is given by

$$
\hat{H}_{\mathrm{s}}=\hat{H}_{\mathrm{L}} \otimes \hat{I}_{\mathrm{R}}-\hat{I}_{\mathrm{L}} \otimes \hat{H}_{\mathrm{R}}^{\mathrm{T}},
$$

where operators with subscript " $L$ " and "R" respectively stand for operators acting on the left and the right systems, and " $T$ " stands for the transpose, and $\hat{I}$ represents the identity operator. $-i \hat{H}_{\mathrm{d}}$ is the non-Hermitian part of the Hamiltonian determined by the dissipation operators, and it is given by

$$
\begin{aligned}
\hat{H}_{\mathrm{d}}= & \sum_{\mu} \gamma_{\mu}\left[-2 \hat{L}_{\mu, \mathrm{L}} \otimes \hat{L}_{\mu, \mathrm{R}}^{*}\right. \\
& \left.+\left(\hat{L}_{\mu}^{\dagger} \hat{L}_{\mu}\right)_{\mathrm{L}} \otimes \hat{I}_{\mathrm{R}}+\hat{I}_{\mathrm{L}} \otimes\left(\hat{L}_{\mu}^{\dagger} \hat{L}_{\mu}\right)_{\mathrm{R}}^{*}\right],
\end{aligned}
$$

where the superscript $*$ stands for taking complex conjugation. We consider that this non-Hermitian Hamiltonian $\hat{H}_{\mathrm{s}}-$ $i \hat{H}_{\mathrm{d}}$ can be diagonalized, and this gives a set of eigenstates that satisfy

$$
\left(\hat{H}_{\mathrm{s}}-i \hat{H}_{\mathrm{d}}\right)\left|\Psi_{\rho}^{l}\right\rangle=\epsilon_{l}\left|\Psi_{\rho}^{l}\right\rangle,
$$

where $\epsilon_{l}$ is a complex number in general. We denote them as $\epsilon_{l}=\alpha_{l}-i \beta_{l}$. This spectrum, originated from the Lindblad equation, is referred to as the Lindblad spectrum. The full Lindblad spectrum has been studied for a number of models before [17-32]. Here we would like to make several useful comments on the Lindblad spectrum. (i) $\alpha_{l}$ and $-\alpha_{l}$ always appear in pairs in the spectrum; (ii) $\beta_{l}$ is always non-negative; and (iii) there always exists at least one zero-energy eigenstate with $\epsilon_{l}=0$, and we label it as $l=0$. Moreover, if $\hat{L}_{\mu}$ are all Hermitian, this steady state is given by $\left|\Psi_{\rho}^{l=0}\right\rangle=$ $\frac{1}{\sqrt{\mathcal{D}_{\mathrm{H}}}} \sum_{n}|n\rangle \otimes|n\rangle$.

\section{RÉNYI ENTROPY AND LINDBLAD SPECTRUM}

Here we consider the second Rényi entropy for the following two reasons. First, it shares the same behavior as the von Neumann entropy and is experimentally accessible [33-35]. Moreover, there exists a close relationship between the dynamics of the second Rényi entropy and the Lindblad spectrum, as we will bring out below. For any density matrix $\hat{\rho}(t)$, the second Rényi entropy $S^{(2)}(t)$ is given by

$$
e^{-S^{(2)}}=\operatorname{Tr}\left(\hat{\rho}^{2}\right)=\sum_{m n} \rho_{m n}(t) \rho_{n m}(t) .
$$

On the other hand, in the double system, the total amplitude of the wave function is given by

$$
\left|\Psi_{\rho}\right|^{2}=\sum_{m n} \rho_{m n}(t) \rho_{m n}^{*}(t)
$$

Since the density matrix is always Hermitian, it gives $\rho_{n m}(t)=\rho_{m n}^{*}(t)$, and therefore we have

$$
e^{-S^{(2)}}=\left|\Psi_{\rho}\right|^{2}
$$


An initial state $\Psi_{\rho}(0)$ in the double space can be expanded as $\Psi_{\rho}(0)=\sum_{l} c_{l}\left|\Psi_{\rho}^{l}\right\rangle$, then its subsequent evolution is given by

$$
\Psi(t)=e^{-i \hat{H}_{\mathrm{s}} t-\hat{H}_{\mathrm{d}} t}\left|\Psi_{\rho}(0)\right\rangle=\sum_{l} c_{l} e^{-i \alpha_{l} t-\beta_{l} t}\left|\Psi_{\rho}^{l}\right\rangle,
$$

and therefore

$$
e^{-S^{(2)}}=\left|\Psi_{\rho}\right|^{2}=\sum_{n}\left|c_{l}\right|^{2} e^{-2 \beta_{l} t}+\cdots
$$

The $\cdots$ term in Eq. (10) represents the terms due to the nonorthogonality condition $\left\langle\Psi_{\rho}^{l^{\prime}} \mid \Psi_{\rho}^{l}\right\rangle \neq 0$ when $l \neq$ $l^{\prime}$, because $\left|\Psi_{\rho}^{l}\right\rangle$ are the eigenstates of a non-Hermitian Hamiltonian. Here we ignore these terms because these nonorthogonality are usually insignificant in the strong dissipation limit.

Since the evolution in the double system is nonunitary and all $\beta_{l}$ are non-negative, the total amplitude of the wave function always decays over time. Hence, by this entropyamplitude relation Eq. (8), the decaying of $\left|\Psi_{\rho}\right|^{2}$ gives rise to the increasing of $S^{(2)}$. Note that for any initial density matrix with trace unity and for Hermitian $\hat{L}_{\mu}, c_{l=0}$ always equals $1 / \sqrt{\mathcal{D}_{\mathrm{H}}}$. This mode always does not decay in time because $\beta_{l=0}=0$. If there are no other eigenstates with $\beta_{l}=0, l=0$ mode is the only remaining mode at an infinitely long time, and this gives a maximum second Rényi entropy $\log \mathcal{D}_{\mathrm{H}}$. Before reaching that limit, the imaginary parts of the Lindblad spectrum of occupied states determine the timescales of the Rényi entropy dynamics. Our discussion below will be based on this connection.

\section{EXAMPLE}

Although the discussion below is quite general for quantum many-body systems, we illustrate the results with two concrete models. The first model is the Bose-Hubbard model which reads

$$
\hat{H}=-J \sum_{\langle i j\rangle}\left(\hat{b}_{i}^{\dagger} \hat{b}_{j}+\text { H.c. }\right)+\frac{U}{2} \sum_{i} \hat{n}_{i}\left(\hat{n}_{i}-1\right),
$$

where $\hat{b}_{i}$ is the boson annihilation operator at site $i$, and $\hat{n}_{i}=\hat{b}_{i}^{\dagger} \hat{b}_{i}$ is the boson number operator at site $i$. $\langle i j\rangle$ denotes nearest neighbor sites. $J$ and $U$ are, respectively, the hopping and the on-site interaction strengths. For the second model we consider hard-core bosons which prevent two bosons to occupy the same site. Also, we introduce the nearest-neighbor repulsion, and the model reads

$$
\hat{H}=-J \sum_{\langle i j\rangle}\left(\hat{b}_{i}^{\dagger} \hat{b}_{j}+\text { H.c. }\right)+V \sum_{\langle i j\rangle} \hat{n}_{i} \hat{n}_{j} .
$$

In one dimension these two models are quite different, because the second model can be mapped to a spinless fermion model with the nearest neighbor repulsion, and can also be mapped to a spin model with the nearest neighbor couplings, but the first model cannot. In both cases, we take all $\hat{n}_{i}$ as the dissipation operators and we set the dissipation strengths uniformly as $\gamma$. In the numerical results shown below, we have chosen $J \sim U$ or $J \sim V$ such that $J$ sets the typical energy scale of the Hamiltonian part, and therefore the strong and weak dissipation, respectively, mean $\gamma / J>1$ or $\gamma / J<1$.

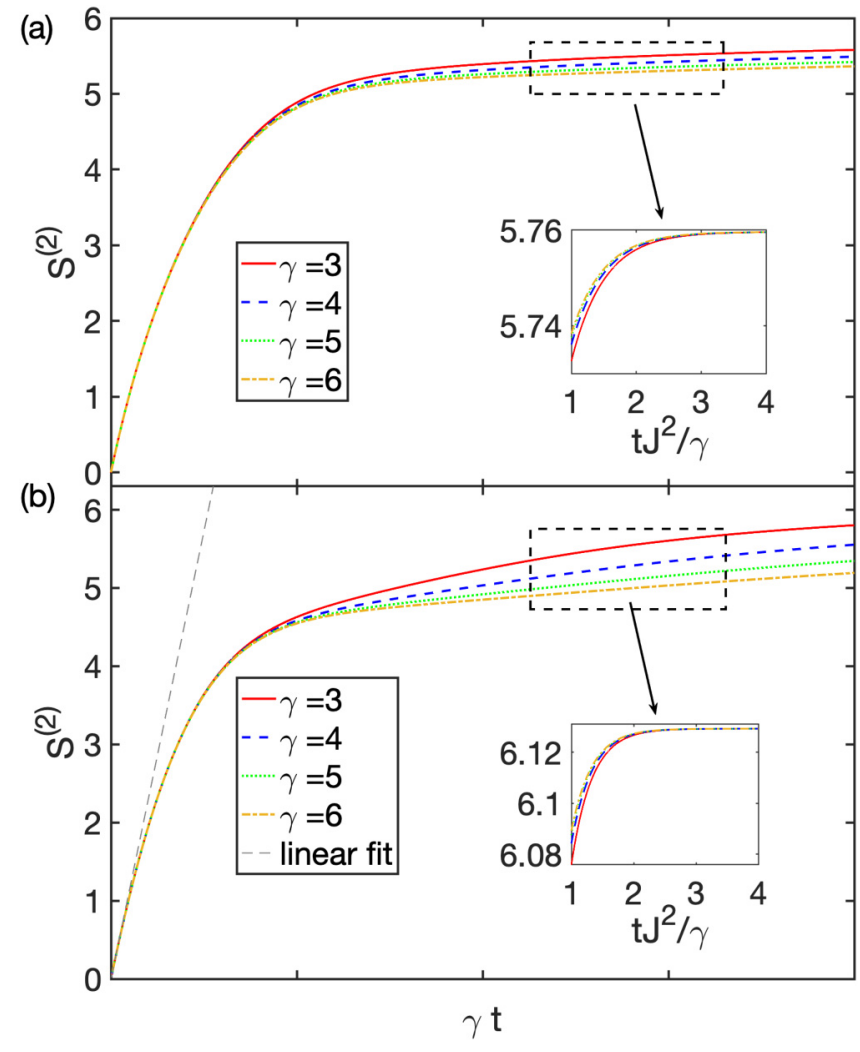

FIG. 2. The dynamics of the second Rényi entropy $S^{(2)}$ as a function of $t \gamma \cdot \gamma$ is the dissipation strength. Different curves have different $\gamma$ in the unit of $J$. The inset shows the long-time behavior of $S^{(2)}$ (indicated by the dashed box) as functions of $t J$ and $t J^{2} / \gamma$. The thinner dashed line shows the initial slop given by Eq. (13). (a) The Bose-Hubbard model with $U=J$ and the number of sites $L=6$, and the number of bosons $N=3$. (b) Hard-core boson model with $V=J, L=8$, and $N=4$. The initial state is the ground state of $\hat{H}$.

\section{DYNAMICS OF THE RÉNYI ENTROPY}

We first consider the short-time behavior of the Rényi entropy dynamics. We apply the short-time expansion to Eq. (9) and utilize the relation Eq. (8), and to the leading order of entropy change we obtain

$$
\lim _{t \rightarrow 0} \frac{d S^{(2)}}{d t}=2 \frac{\left\langle\Psi_{\rho}(0)\left|\hat{H}_{\mathrm{d}}\right| \Psi_{\rho}(0)\right\rangle}{\left\langle\Psi_{\rho}(0) \mid \Psi_{\rho}(0)\right\rangle} .
$$

The physical meaning of the r.h.s. of Eq. (13) in the original system is the fluctuation of the dissipation operators. For instance, if the initial state is a pure state denoted as $\hat{\rho}(0)=$ $|\psi(0)\rangle\langle\psi(0)|$, then we have $\left|\Psi_{\rho}(0)\right\rangle=|\psi(0)\rangle \otimes|\psi(0)\rangle$ according to it. Then, Eq. (13) can be rewritten as

$$
\begin{aligned}
\lim _{t \rightarrow 0} \frac{d S^{(2)}}{d t}= & 4 \sum_{\mu} \gamma_{\mu}\left(\left\langle\psi(0)\left|\hat{L}_{\mu}^{\dagger} \hat{L}_{\mu}\right| \psi(0)\right\rangle\right. \\
& \left.-\left|\left\langle\psi(0)\left|\hat{L}_{\mu}\right| \psi(0)\right\rangle\right|^{2}\right) .
\end{aligned}
$$

Suppose all $\gamma_{\mu}$ are taken as the same $\gamma$, this result shows that the time dependence of $S^{(2)}$ is governed by a dimensionless time $\gamma t$. In another word, the larger $\gamma$ is, the faster the Rényi entropy increases. This $\gamma t$ scaling is shown in Fig. 2 where one can see that the short-time parts of the $S^{(2)}$ curves with 

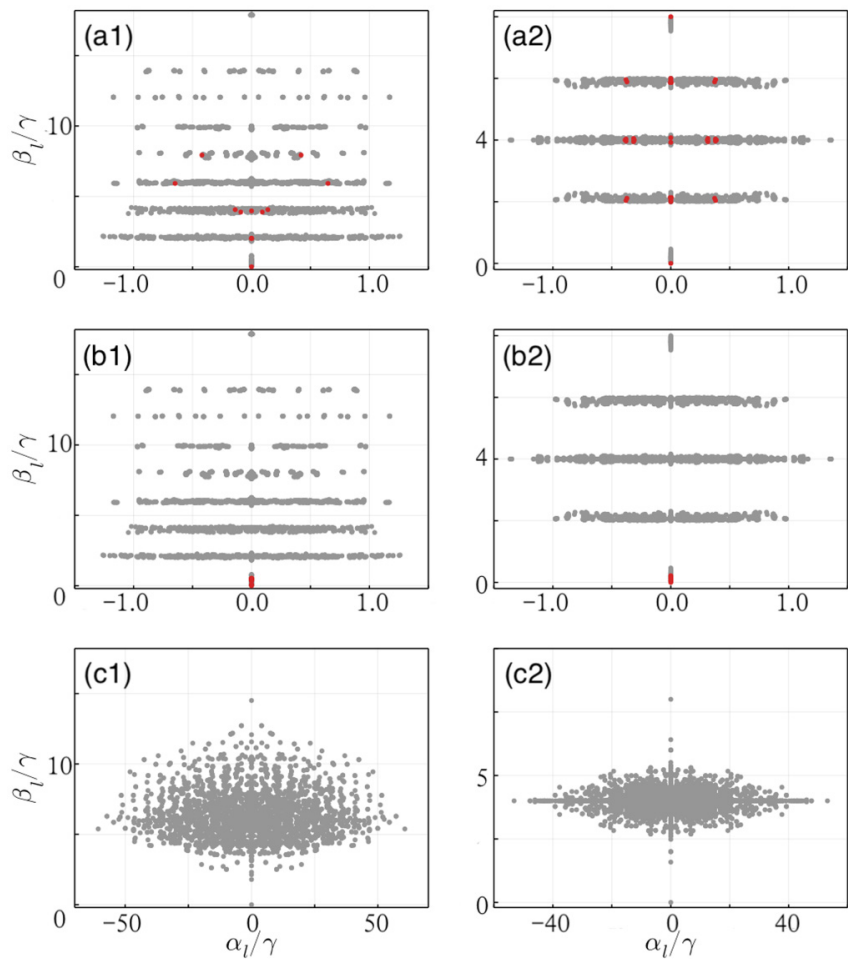

FIG. 3. The Lindblad spectrum for strong dissipation case (a1), (a2), (b1), and (b2) with $\gamma=5 J$ and for weak dissipation case (c1) and (c2) with $\gamma=0.2 J$. The red points mark the eigenstates with significant occupation $\left(\left|c_{l}\right|^{2} \geqslant 1 / \mathcal{D}_{\mathrm{H}}\right)$ by the initial state. For (a1) and (a2) in the first row, the initial state is taken as $\Psi_{\rho}=\left|\psi_{\mathrm{g}}\right\rangle \otimes\left|\psi_{\mathrm{g}}\right\rangle$, where $\left|\psi_{\mathrm{g}}\right\rangle$ is the ground state of $\hat{H}$. For (b1) and (b2) in the second row, the initial states are taken as the zero-energy eigenstate of $\hat{H}_{\mathrm{d}}$ that are $|111000\rangle$ for (b1) and $|11110000\rangle$ for (b2) in Fock bases. The left column (a1), (b1), and (c1) are for the Bose-Hubbard model with $U=J$ and the number of sites $L=6$, and the number of bosons $N=3$. The right column (a2), (b2), and (c2) are for hard core boson model with $V=J, L=8$, and $N=4$.

different $\gamma$ collapse into a single line when they are plotted in terms of $\gamma t$. The thinner dashed line in Fig. 2 compares the short-time behavior of $S^{(2)}$ with the slope given by Eqs. (13) and (14). From Fig. 2 one can also find that $S^{(2)}$ no longer obeys the $\gamma t$ scaling when $\gamma t>1$. Moreover, in the strong dissipation regime, the inset plotted in term of $t J$ shows an opposite trend at long time, that is, the larger $\gamma$ is, the slower the Rényi entropy increases. In fact, the long-time behavior of $S^{(2)}$ exhibits a $t / \gamma$ scaling. As shown in the inset of Fig. 2, when the long-time part of $S^{(2)}$ curves with different $\gamma$ are plotted in terms of $t J^{2} / \gamma$, they all collapse into a single curve.

\section{LINDBLAD SPECTRUM WITH STRONG DISSIPATION}

These opposite behaviors between the short- and the longtime entropy dynamics can be understood very well in terms of the Lindblad spectrum. As one can see from Figs. 3(a) and 3(b), for the strong dissipation, the main feature of the Lindblad spectrum is that it separates into segments along the imaginary axes of the spectrum, and the separations between segments are approximately $2 \gamma$. For each segment, the width along the imaginary axes is approximately given by $J^{2} / \gamma$.

Although we show this feature with our concrete models, the physical picture can be understood by general perturbation treatment of $\hat{H}_{\mathrm{s}}-i \hat{H}_{\mathrm{d}}$. First, since the dissipation strength is stronger than the typical energy scales of the Hamiltonian, we can treat $\hat{H}_{\mathrm{s}}$ as a perturbation to $\hat{H}_{\mathrm{d}}$. To the zeroth order of $\hat{H}_{\mathrm{d}}$, the spectrum is purely imaginary, and different segments are separated by the order of the dissipation strength. Second, when the dissipation term is a sum of local operators, the eigenstates of $\hat{H}_{\mathrm{d}}$ are usually highly degenerate because different $\hat{L}_{\mu}$ commute with each other and share the same set of eigenvalues, such as $\hat{L}_{\mu}$ being $\hat{n}_{i}$ in our examples. Third, $\hat{H}_{\mathrm{s}}$ and $\hat{H}_{\mathrm{d}}$ usually do not commute with each other, and the perturbation in $\hat{H}_{\mathrm{s}}$ lifts the degeneracy of the imaginary parts and gives rise to a broadening due to the nature of the second-order perturbation. However, the nature of second-order perturbation ensures that the broadening is much smaller compared with the separation of different segments.

Let us call the eigenstates with imaginary parts of the order of a few times of $\gamma$ as "high imaginary energy states," and the eigenstates with imaginary parts of the order of $J^{2} / \gamma$ as "lowlying imaginary energy states." For a generic initial state, both two types of eigenstates are occupied. Quite generally, the occupations of the high imaginary energy states are significant, for instance, when the initial state is the eigenstates of $\hat{H}_{\mathrm{s}}$. With the relation between the Rényi entropy dynamics and the Lindblad spectrum discussed above, it is clear that the shorttime dynamics is dominated by these high imaginary energy states that gives rise to a dynamics scaled by $t \gamma$. Nevertheless, when $\gamma t>1$, the weights on these high imaginary energy states almost decay out, and long-time dynamics is therefore dominated by the low-lying imaginary energy states that gives rise to a dynamics scaled by $t J^{2} / \gamma$.

\section{QUANTUM ZENO EFFECT REVISITED}

Here we consider a specific initial state that satisfies $\hat{H}_{\mathrm{d}}|\Psi(0)\rangle=0$. In another word, such initial states do not exhibit fluctuation of dissipation operators. Thus, according to Eqs. (13) and (14), the initial slop of $S^{(2)}$ is zero. Moreover, in the strong dissipation regime, the populations of the high imaginary energy states are strongly suppressed by the gap between different segments and their contribution becomes negligible. Such initial states mainly populate the low-lying imaginary energy states, as we have shown in Fig. 3(b). Therefore, the entire dynamics of the second Rényi entropy is set by the energy scale $J^{2} / \gamma$, and it obeys the $t / \gamma$ scaling. This is shown in Fig. 4 for two models. To contrast such specific initial states with generic states discussed above, we plot the short-time behavior of $S^{(2)}$ as a function of $t \gamma$ and $t J$ in the inset of Fig. 4. Unlike the results shown in Fig. 2, the short-time dynamics with $t \gamma<1$ does not exhibit the linear behavior and different curves do not collapse into a single line in terms of $t \gamma$.

For these initial states, the dynamics are slower with stronger dissipation is reminiscent of the quantum Zeno effect [3-6]. In fact, the quantum Zeno effect can be understood in this way. Introducing $\{|M\rangle\}\left(M=1, \ldots, \mathcal{D}_{\mathrm{H}}\right)$, as a set of 


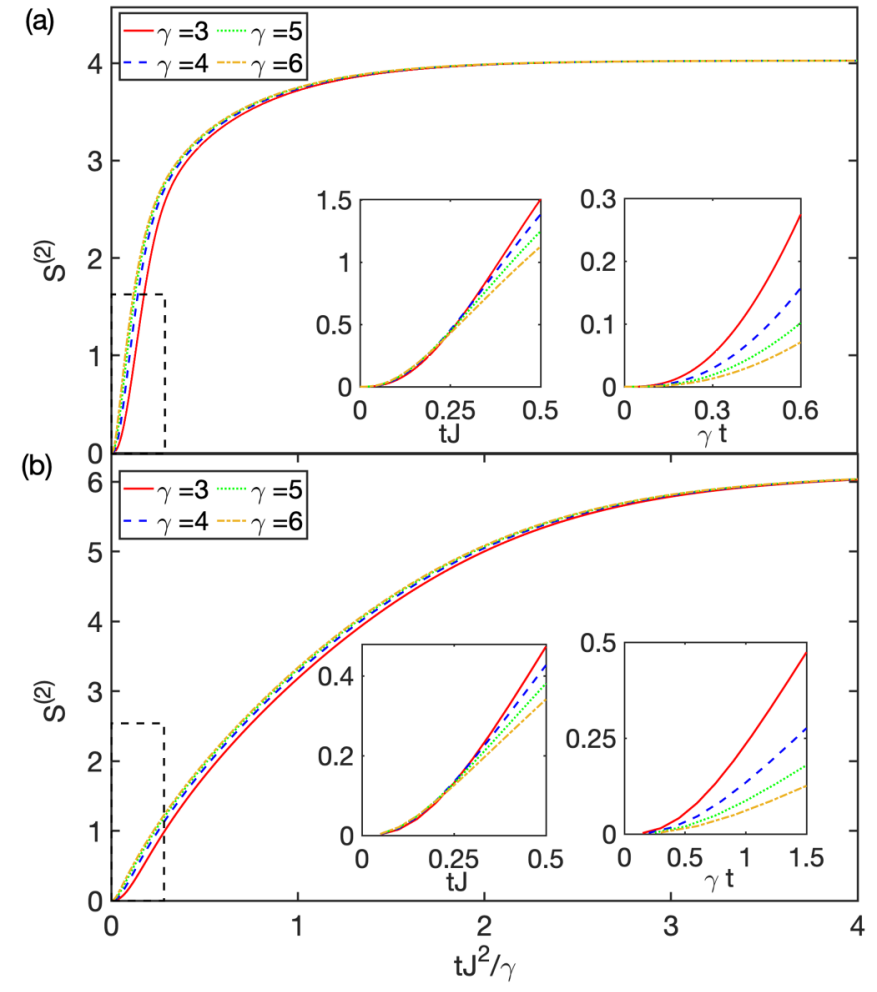

FIG. 4. The dynamics of the second Rényi entropy $S^{(2)}$ as a function of $t J^{2} / \gamma$ for a specific initial state. $\gamma$ is the dissipation strength. Different curves have different $\gamma$ in the unit of $J$. The inset shows the short-time behavior of $S^{(2)}$ (indicated by the dashed box) as functions of $t J$ and $t \gamma$. (a) The Bose-Hubbard model with $U=J$ and the number of sites $L=6$, and the number of bosons $N=3$. (b) Hard-core boson model with $V=J, L=8$, and $N=4$. The initial states are taken as the zero-energy eigenstate of $\hat{H}_{\mathrm{d}}$ that are $|111000\rangle$ for (a) and $|11110000\rangle$ for (b) in Fock bases.

complete and orthogonal measurement bases, we define the projection operators as $\hat{P}_{M}=|M\rangle\langle M|$. The frequent measurement process can also be described by the Lindblad master equation (1) with the dissipation operator $\hat{L}_{\mu}$ given by all $\hat{P}_{M}$. With such dissipation operators, the Lindblad spectrum exhibits a set of low-lying imaginary energy states with an energy scale given by $J^{2} / \gamma$. It can be shown that, as long as the initial state density matrix is diagonal in the measurement bases, the initial states satisfy $\hat{H}_{\mathrm{d}}|\Psi(0)\rangle=0$.

\section{FROM STRONG TO WEAK DISSIPATION}

Finally, we show that when $\gamma$ decreases and eventually becomes weaker compared with the typical energy scales in the Hamiltonian, the segment structure in the Lindblad spectrum disappears, as we have shown in Fig. 3(c). Thus, the entropy dynamics for generic states no longer displays the feature of two timescales. The quantum Zeno effect also disappears for any specific initial state. This is understandable because the typical time interval between two measurements is already longer than the intrinsic evolution time of the system in this regime.

\section{SUMMARY}

In this work we establish a relation between the Rényi entropy dynamics and the Lindblad spectrum in double space. At the strong dissipation regime, the Lindblad spectrum exhibits a segment structure, in which we can introduce the high imaginary energy eigenstates and the low-lying imaginary energy eigenstates. For a generic initial state with significantly occupied high imaginary energy eigenstates, the former dominates the short-time dynamics and the latter dominates the long-time dynamics, and them respectively give rise to $t \gamma$ scaling and $t / \gamma$ scaling. For a specific initial state with only low-lying imaginary energy eigenstates significantly occupied, the dynamics are dominated by $t / \gamma$ scaling. The quantum Zeno effect belongs to this class. We illustrate our results with two concrete models. The second Rényi entropy can now be measured in ultracold atomic gases in optical lattices. In fact, it has been measured in the Bose-Hubbard model with or without disorder [33-35]. The dissipation operators and their strengths can also be controlled in ultracold atomic gases [36]. Our predictions can therefore be verified directly in the experimental setup.

Note added. When finishing this work, we become aware of a work in which similar behaviors of the Lindblad spectrum in the strong dissipation regime are also discussed [37].

\section{ACKNOWLEDGMENTS}

We thank Lei Pan, Tian-Shu Deng, Tian-Gang Zhou, Pengfei Zhang, and He-Ran Wang for helpful discussions. This work is supported by Beijing Outstanding Young Scientist Program, NSFC Grant No. 11734010, MOST under Grant No. 2016YFA0301600.
[1] H. P. Breuer and F. Petruccione, The Theory of Open Quantum Systems (Oxford University Press, Oxford, 2007).

[2] L. Pan, X. Chen, Y. Chen, and H. Zhai, Non-Hermitian linear response theory, Nat. Phys. 16, 767 (2020).

[3] B. Misra and E. C. G. Sudarshan, The Zeno's paradox in quantum theory, J. Math. Phys. 18, 756 (1977).

[4] W. M. Itano, D. J. Heinzen, J. J. Bollinger, and D. J. Wineland, Quantum Zeno effect, Phys. Rev. A 41, 2295 (1990).

[5] A. G. Kofman and G. Kurizki, Acceleration of quantum decay processes by frequent observations, Nature (London) 405, 546 (2000).
[6] K. Koshino and A. Shimizu, Quantum Zeno effect by general measurements, Phys. Rep. 412, 191 (2005).

[7] L. Aolita, F. de Melo, and L. Davidovich, Open-system dynamics of entanglement: A key issues review, Rep. Prog. Phys. 78, 042001 (2015).

[8] L. Piroli, C. Sünderhauf, and X.-L. Qi, A random unitary circuit model for black hole evaporation, J. High Energy Phys. 04 (2020) 63.

[9] Y. Chen, X.-L. Qi, and P. Zhang, Replica wormhole and information retrieval in the SYK model coupled to Majorana chains, J. High Energy Phys. 06 (2020) 121. 
[10] V. Alba and F. Carollo, Spreading of correlations in Markovian open quantum systems, Phys. Rev. B 103, L020302 (2021).

[11] P. Dadras and A. Kitaev, Perturbative calculations of entanglement entropy, J. High Energy Phys. 03 (2021) 198.

[12] Y. Chen, Entropy linear response theory with non-Markovian bath, J. High Energy Phys. 04 (2021)215.

[13] K. Su, P. Zhang, and H. Zhai, Page curve from nonMarkovianity, J. High Energy Phys. 06 (2021) 156.

[14] J. E. Tyson, Operator-Schmidt decompositions and the Fourier transform, with applications to the operator-Schmidt numbers of unitaries, J. Phys. A: Math. Gen. 36, 10101 (2003).

[15] M. Zwolak and G. Vidal, Mixed-State Dynamics in OneDimensional Quantum Lattice Systems: A Time-Dependent Superoperator Renormalization Algorithm, Phys. Rev. Lett. 93, 207205 (2004).

[16] T. Prosen, Third quantization: A general method to solve master equations for quadratic open Fermi systems, New J. Phys. 10, 043026 (2008).

[17] T. Prosen, $\mathcal{P} \mathcal{T}$-Symmetric Quantum Liouvillean Dynamics, Phys. Rev. Lett. 109, 090404 (2012).

[18] M. Žnidarić, Relaxation times of dissipative many-body quantum systems, Phys. Rev. E 92, 042143 (2015).

[19] M. V. Medvedyeva, F. H. L. Essler, and T. Prosen, Exact Bethe Ansatz Spectrum of a Tight-Binding Chain with Dephasing Noise, Phys. Rev. Lett. 117, 137202 (2016).

[20] S. Denisov, T. Laptyeva, W. Tarnowski, D. Chruciski, and K. Zyczkowski, Universal Spectra of Random Lindblad Operators, Phys. Rev. Lett. 123, 140403 (2019).

[21] T. Can, V. Oganesyan, D. Orgad, and S. Gopalakrishnan, Spectral Gaps and Midgap States in Random Quantum Master Equations, Phys. Rev. Lett. 123, 234103 (2019).

[22] S. Lieu, M. McGinley, and N. R. Cooper, Tenfold Way for Quadratic Lindbladians, Phys. Rev. Lett. 124, 040401 (2020).

[23] K. Wang, F. Piazza, and D. J. Luitz, Hierarchy of Relaxation Timescales in Local Random Liouvillians, Phys. Rev. Lett. 124, 100604 (2020).

[24] D. Yuan, H. Wang, Z. Wang, and D. L. Deng, Solving the Liouvillian Gap with Artificial Neural Networks, Phys. Rev. Lett. 126, 160401 (2021).
[25] T. Can, Random Lindblad dynamics, J. Phys. A: Math. Theor 52, 485302 (2019).

[26] L. Sa, P. Ribeiro, and T. Prosen, Spectral and steady-state properties of random Liouvillians, J. Phys. A: Math. Theor. 53, 305303 (2020).

[27] O. E. Sommer, F. Piazza, and D. J. Luitz, Many-body hierarchy of dissipative timescales in a quantum computer, Phys. Rev. Res. 3, 023190 (2021)

[28] W. Tarnowski, I. Yusipov, T. Laptyeva, S. Denisov, D. Chruscinski, and K. Zyczkowski, Random generators of Markovian evolution: A quantum-classical transition by superdecoherence, Phys. Rev. E 104, 034118 (2021).

[29] D. A. Rowlands and A. Lamacraft, Noisy Spins and the Richardson-Gaudin Model, Phys. Rev. Lett. 120, 090401 (2018).

[30] P. Ribeiro and T. Prosen, Integrable Quantum Dynamics of Open Collective Spin Models, Phys. Rev. Lett. 122, 010401 (2019).

[31] N. Shibata and H. Katsura, Dissipative quantum Ising chain as a non-Hermitian Ashkin-Teller model, Phys. Rev. B 99, 224432 (2019).

[32] N. Shibata and H. Katsura, Dissipative spin chain as a nonHermitian Kitaev ladder, Phys. Rev. B 99, 174303 (2019).

[33] R. Islam, R. Ma, P. M. Preiss, M. Eric Tai, A. Lukin, M. Rispoli, and M. Greiner, Measuring entanglement entropy in a quantum many-body system, Nature (London) 528, 77 (2015).

[34] A. M. Kaufman, M. Eric Tai, A. Lukin, M. Rispoli, R. Schittko, P. M. Preiss, and M. Greiner, Quantum thermalization through entanglement in an isolated many-body system, Science 353, 794 (2016).

[35] A. Lukin, M. Rispoli, R. Schittko, M. E. Tai, A. M. Kaufman, S. Choi, V. Khemani, J. Léonard, and M. Greiner, Probing entanglement in a many-body-localized system, Science 364, 256 (2019).

[36] R. Bouganne, M. B. Aguilera, A. Ghermaoui, J. Beugnon, and F. Gerbier, Anomalous decay of coherence in a dissipative many-body system, Nat. Phys. 16, 21 (2020).

[37] V. Popkov and C. Presilla, Full Spectrum of the Liouvillian of Open Dissipative Quantum Systems in the Zeno Limit, Phys. Rev. Lett. 126, 190402 (2021). 